\title{
Diversidad y composición de Odonata asociadas con diferentes condiciones de la vegetación riparia a lo largo de un arroyo en Paicol-Huila, Colombia
}

\author{
Odonata diversity and composition associated with different conditions in riparian vegetation \\ along a stream located in Colombia
}

\begin{abstract}
José A. Cuéllar-Cardozo ${ }^{1,2 *}$, María I. Castro-Rebolledo ${ }^{2}$, M. Alejandra Jaramillo ${ }^{1}$
Resumen

El proceso de fragmentación, a consecuencia de las actividades humanas, ha modificado la diversidad de los ecosistemas acuáticos y riparios, especialmente en áreas como bosques secos tropicales que, en Colombia, son zonas en peligro de desaparecer dentro de pocos años. Ante esta situación, la diversidad y la composición de los insectos Odonata, que están influenciadas por factores abióticos, podrían ser excelentes herramientas para comprender el estado ambiental actual. Por lo anterior, el objetivo de este estudio fue observar los cambios en la diversidad y la composición de Odonata, en diferentes condiciones de la vegetación riparia, a lo largo de un arroyo, en un remanente de bosque seco. Al mismo tiempo, se plantea la hipótesis de que existen diferencias, en la diversidad y composición de Odonata, a lo largo del sistema. Para esto, se muestrearon larvas y adultos de odonatos y se determinaron las variables ambientales a lo largo de cuatro estaciones de muestreo dentro de un mismo arroyo, con diferente vegetación ribereña, en cuatro periodos durante un año. Se recolectó un total de 272 especímenes correspondientes a 16 especies. Se observaron variaciones en la composición de Odonata, señaladas en los registros de especies y en el índice de similitud. Sin embargo, no se observaron diferencias significativas en la diversidad de odonatos. En cuanto a la fisicoquímica del arroyo, se notó una variación espacial en la conductividad y la concentración de nutrientes y una variación temporal en el pH y la temperatura. A manera de conclusión, se exponen cambios en la composición de Odonata, en concordancia con la vegetación riparia, durante el año de muestreo.
\end{abstract}

Palabras claves: Bosque Seco Tropical, caballitos del diablo, libélulas, Huila, región Andina

\begin{abstract}
The fragmentation of aquatic and riparian environments has damaged diversity into ecosystems. These injuries are pronounced in areas as tropical dry forests in Colombia, which are endangered zones that could be disappear in few years. In this situation, diversity and composition in Odonata insects, which are influenced by abiotic factors, could be excellent tools for understanding environmental status. Thus, we aim to observe changes in Odonata diversity and composition in different riparian vegetation during a year, along a stream from a remnant of dry forest. At the same time, we hypothesize that there are differences in Odonata diversity and composition. To take this, we sampled Odonata, larvae and adults, and environmental variables in four sampling stations, with different riparian vegetation during a year. We collected a total of 272 specimens corresponding to 16 species. In one hand, we noted variations in Odonata composition, showed in species records and similarity index. In other hand, we do not observe differences in Odonata diversity. Lastly, we approve in part our hypothesis exposing only Odonata composition changes with riparian vegetation, during sampling year.
\end{abstract}

Keywords: Tropical Dry Forest, dragonflies, damselflies, Huila, Andean region

\footnotetext{
1. DIVERSITAS, Universidad Militar Nueva Granada, Cajicá-Zipaquirá, Colombia.

2. Bioprospección y Biodiversidad Colombiana, Universidad La Salle, Bogotá. Colombia.

* Autor de correspondencia: <josecuellar1094@gmail.com>
} 


\section{INTRODUCCIÓN}

El aumento de las actividades agropecuarias y de zonas urbanas afectan directamente los ecosistemas riparios y acuáticos de Colombia y el mundo (CarvajalCogollo, 2008; Valdés, 2011). Esta intervención humana en el paisaje natural ha producido fenómenos como la degradación progresiva del hábitat, generando fragmentos de vegetación nativa rodeada de nuevos tipos de ambientes como cultivos, en un proceso conocido como fragmentación del ecosistema (Montenegro, 2009; Valdés, 2011; Williams-Linera, Manson, y Isunza, 2002). En los ecosistemas riparios y acuáticos, el proceso de fragmentación ha causado la pérdida de vegetación ribereña y cambios en la composición de macrófitas, alterando la entrada de energía alóctona al ecosistema acuático (Moulton, 2006; Neres-Lima et al, 2017). De manera similar, este proceso tiene un efecto particular en las áreas del bosque seco tropical (BST), afectadas por su importancia agrícola, a consecuencia de sus suelos llenos de nutrientes, causando su fragmentación, reducción y desertificación (Miles et al, 2006). Finalmente, este proceso de fragmentación ha impulsado la reducción en las áreas de BST en Colombia, que actualmente ocupan menos de $85.000 \mathrm{~km}^{2}$, cerca del $8 \%$ del territorio del país y menos del $10 \%$ de su área potencial (Gómez, Moreno, Andrade, y Rueda, 2016; Pizano y Garcia, 2014).

Al mismo tiempo, el proceso de fragmentación ha provocado alteraciones en los ambientes dulceacuícolas, promoviendo el cambio en la diversidad de macroinvertebrados y la capacidad de recuperación del hábitat (Feld et al, 2016; Firmiano et al, 2017). Esta modificación de la diversidad de los macroinvertebrados se relaciona con alteraciones en los procesos ecosistémicos como el mantenimiento de la energía de las redes alimentarias en los entornos acuáticos y riparios (Hepp y Santos, 2009; Johnson, Jin, Carreiro, y Jack, 2013). El orden Odonata está altamente implicado en este proceso energético, ya que algunas especies son muy dependientes de las condiciones ecológicas de las áreas acuáticas y las poblaciones de otros macroinvertebrados, lo que nos permite usarlo como medio para comprender los cambios que ocurren dentro de los ecosistemas acuáticos y riparios (Benke, Huryn, Smock, y Wallace, 1999; Corbet,
2004). De esta manera, la diversidad y la composición de odonatos pueden emplearse como indicadores del grado de perturbación en los ecosistemas acuáticos y riparios afectados por la fragmentación y la influencia antropogénica (Monteiro-Júnior, Couceiro, Hamada, y Juen, 2013). En los últimos años, muchos investigadores han promovido el uso de odonatos como indicadores de perturbaciones ambientales, especialmente en el Neotrópico (Monteiro-Júnior et al, 2013; Silva, De Marco, y Resende, 2010; Tognelli, Lasso, Bota-Sierra, Jiménez-Segura, y Cox, 2016). No obstante, en Colombia las investigaciones sobre Odonata se han centrado en estudios taxonómicos extensos, y aún faltan investigaciones que promuevan a los odonatos como una herramienta en la restauración y conservación ambiental (McGeogh, 1997; Palacino-Rodríguez, 2016).

En efecto, comprender los cambios en la biodiversidad ayuda a inferir el proceso de fragmentación y el grado de perturbación antropogénica dentro de los ecosistemas riparios y acuáticos, especialmente en zonas amenazadas como el BST. Por lo tanto, el objetivo de este artículo fue observar los cambios en la diversidad y la composición de Odonata en áreas con diferentes tipos de vegetación riparia, en cuatro periodos de un año, a lo largo de un arroyo ubicado en un remanente de BST en Paicol, Huila (Colombia). Además, se planteó la hipótesis de que existen diferencias significativas en la diversidad y la composición de Odonata, y que estas se relacionan con las diferencias en la vegetación riparia a lo largo del arroyo y los períodos de muestreo que corresponden a las temporadas de lluvia y sequía.

\section{MATERIALES Y MÉTODOS}

\section{Área de estudio}

Los odonatos se muestrearon a lo largo del arroyo "La Avería", localizado en el municipio de Paicol, departamento de Huila, Colombia. Paicol presenta una temperatura promedio de $27^{\circ} \mathrm{C}$, la precipitación media anual es de $1820 \mathrm{~mm}$, con una estación seca entre los meses de marzo y agosto, y una estación húmeda comprendida entre septiembre y febrero (Instituto de Hidrología, Meteorología y Estudios Ambientales [IDEAM], 2016). El arroyo tiene una longitud 
total de $5 \mathrm{~km}$ aproximadamente, el flujo de agua nace dentro de un remanente conservado de BST y posteriormente cruza áreas donde el bosque seco ha sido perturbado por actividades humanas como los cultivos de arroz o la ganadería, para finalmente desembocar en el río Páez (figura 1).

Se definieron cuatro estaciones de muestreo a lo largo del arroyo separadas por una distancia de aproximadamente un $\mathrm{km}$ entre cada estación de muestreo. Se caracterizó la cubierta del dosel adyacente al arroyo con un muestreo e identificación de la vegetación utilizando la información taxonómica de Vargas (2015). La zona alta está cubierta por bosque nativo (Estación 1: $2^{\circ} 26^{\prime} 22,3^{\prime \prime} \mathrm{N}$ y $75^{\circ} 46^{\prime} 31,4^{\prime \prime} \mathrm{W} \pm 822 \mathrm{~m} \mathrm{~s}$. n. m.) correspondiente a parches preservados de BST interconectados entre ellos. La vegetación riparia en esta estación estuvo compuesta por grandes árboles y arbustos, entre lo que dominan: Clavija sp., Guadua angustifolia, Guarea guidonia, Machaerium capote, Pseudosamanea guachapele y Renealmia sp. La segunda estación de muestreo es una combinación de parches interconectados de bosque nativo y amplias áreas de cría de ganado (Estación 2: $2^{\circ} 26^{\prime} 51,2^{\prime \prime} \mathrm{N}$ y $75^{\circ} 46^{\prime} 11,3^{\prime \prime} \mathrm{W} \pm 819 \mathrm{~m}$ s. n. m.); la vegetación a las orillas del arroyo consistió en una mezcla de diferentes plantas como Adiantum sp., Carludovica palmata, Cenchrus clandestinus y Selaginella sp. La tercera estación estaba dominada por arrozales a lo largo del arroyo, además de ser una zona de vertimientos de aguas residuales provenientes del centro urbano cercano (Estación 3: $2^{\circ} 26^{\prime} 31,8^{\prime \prime} \mathrm{N}$ y $75^{\circ} 46^{\prime} 5,9^{\prime \prime} \mathrm{W}$ $\pm 813 \mathrm{~m}$ s. n. m.). Esta estación presenta vegetación ribereña dominada por Cecropia sp., Piper spp. y macrófitas como Spathiphyllum sp. acompañando los arrozales. En la parte baja del arroyo, la última estación estuvo compuesta por áreas de cría de ganado bovino (Estación 4: $2^{\circ} 26^{\prime} 57,2^{\prime \prime} \mathrm{N}$ y $75^{\circ} 45^{\prime} 24,6^{\prime \prime}$ $\mathrm{W} \pm 805 \mathrm{~m}$ s. n. m.) con campos de hierba de C. clandestinus y macrófitas como Cyperus sp. que fueron abundantes en toda la estación.

\section{Muestreo}

Se realizaron muestreos durante febrero, mayo, agosto y noviembre de 2016, para cubrir todo el ciclo de lluvias del área de estudio. Se midieron: conductividad $\left(\mu \mathrm{s} \mathrm{cm}^{-1}\right)$, oxígeno disuelto $\left(\mathrm{mg} \mathrm{L}^{-1}\right)$, sólidos suspendidos totales $\left(\mathrm{mg} \mathrm{L}^{-1}\right)$, $\mathrm{pH}$, temperatura $\left({ }^{\circ} \mathrm{C}\right)$ y caudal $\left(\mathrm{m}^{3} \mathrm{~s}^{-1}\right)$, utilizando una sonda multiparamétrica marca HANNA ${ }^{\circledR}$. Además, se determinaron las concentraciones $\left(\mathrm{mg} \mathrm{L}^{-1}\right)$ de amonio $\left(\mathrm{NH}_{4}^{+}\right)$, nitrato $\left(\mathrm{NO}_{3}^{-}\right)$y fosfato $\left(\mathrm{PO}_{4}^{-3}\right)$ mediante el análisis colorimétrico propuesto por API Freshwater Master Test Kit. Todas las variables fisicoquímicas e hidrológicas se midieron in situ tres veces por período de muestreo en cada punto de muestreo.

En cada estación se capturaron odonatos adultos

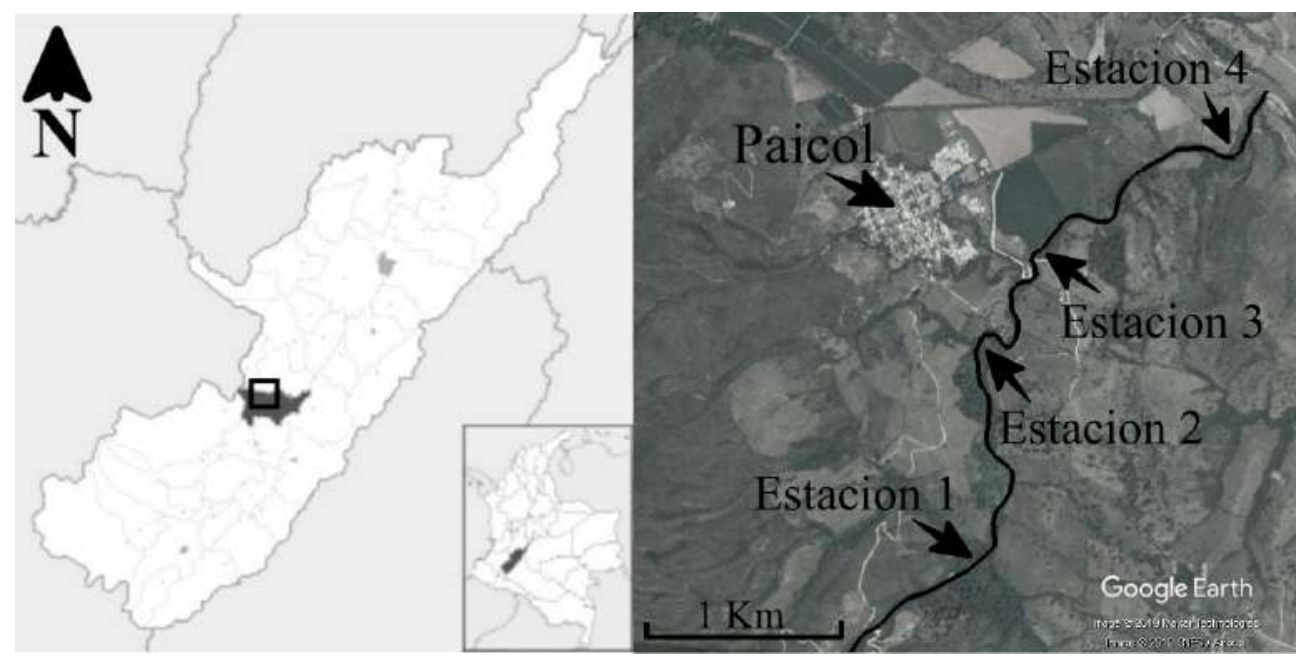

Figura 1. Área de estudio en el departamento del Huila, Colombia. Ubicación de las estaciones de muestreo en el arroyo La Avería. 
por medio de redes entomológicas, en tres transectos lineales de $50 \mathrm{~m}$, perpendiculares al arroyo. Por otro lado, se recolectaron larvas en etapas cercanas a la emergencia, para facilitar su posterior identificación, utilizando una D-net encerrada por un cilindro (diámetro $0,55 \mathrm{~m}$ ) en 10 ubicaciones adyacentes a las macrófitas del arroyo, a 0,3 $\mathrm{m}$ de profundidad en tres transectos de $50 \mathrm{~m}$ en cada estación de muestreo por periodo de muestreo. El proceso de recolecta presentó un esfuerzo de muestreo de $90 \mathrm{~min}$, para larvas y adultos por separado, para cada estación durante cada período de muestreo, eligiendo el momento con la mayor luz solar en el día. Consecutivamente, los organismos se identificaron taxonómicamente a nivel de especie, utilizando las claves disponibles para adultos (De Marmels, 2012; Garrison, 1994; Garrison y Ellenrieder, 2015; Garrison, Ellenrieder, y Louton, 2006; Leonard, 1977; May, 1998; Meurgey y Poiron, 2011; Ris, 1930; von Ellenrieder, 2003; Walker, 2015) y larvas (Belle, 1996; Limongi S, 1983; NoveloGutierrez, 2002; Novelo-Gutiérrez, 2005; Zloty, Pritchard, y Esquivel, 1993). Las identificaciones fueron corroboradas y verificadas por especialistas del orden Odonata, del grupo LAZOEA de la Universidad de los Andes, Bogotá-Colombia. Por último, los especímenes recolectados fueron dispuestos en el Museo La Salle en Bogotá, Colombia.

\section{Análisis estadístico}

Para definir cambios significativos en los datos fisicoquímicos, a lo largo de las estaciones y los periodos de muestreo, se utilizó un ANOVA $(p<0,05)$. Se calcularon las curvas de rango-abundancia de los organismos por estación y periodo de muestreo. Adicionalmente, con el software EstimateS Win v9.1 (Colwell et al, 2012) se estimó si el esfuerzo de muestreo fue efectivo utilizando una curva de rarefacción con la riqueza estimada, el estimador Jacknife de primer orden y el índice Estimador basado en la Cobertura de Abundancia (ACE); en este último índice se consideraron como especies raras a aquellas especies con cinco o menos individuos. Estos índices se usaron porque presentaron el estimador más preciso y menos sesgado en una comparación de varios procedimientos (Palmer, 1990). Posteriormente, se calculó la diversidad $\alpha$, agregando adultos y larvas, por estación de muestreo y tiempo, utilizando el índice diversidad de Shannon-Wiener $\left(\mathrm{H}^{\prime}\right)$ en el programa PAST v3.14 (Hammer, Harper, y Ryan, 2001). Además de utilizar un ANOVA $(p<0,05)$ para encontrar diferencias significativas en la diversidad de los insectos de acuerdo con la estación y periodo de muestreo. Finalmente, para determinar la diversidad $\beta$ entre la estación de muestreo y el tiempo, se usó un análisis de similitud y dendrogramas, a partir del índice de Jaccard, utilizando el algoritmo pairedgroup (Jackson y Somers, 1989).

\section{RESULTADOS}

Se capturaron 272 especímenes, 168 adultos y 104 larvas, comprendidos en cinco familias y 16 especies en toda el área de estudio durante el año de recolección (tabla 1). La curva de rango-abundancia mostró que la Estación 1 tuvo la menor abundancia de individuos (10 adultos y 10 larvas), mientras que la Estación 4 presentó la mayor abundancia de individuos con 82 adultos y 44 larvas; la Estación 2 exhibió la mayor riqueza, con 12 especies (figura 2A). La mayoría de los individuos de Odonata fueron recolectados al comienzo de la estación húmeda, al igual que la mayor riqueza de especies se observó durante la época de noviembre a febrero (figura 2B). Las especies más abundantes fueron Enallagma novaehispaniae (Calvert, 1907) con 67 individuos (32 adultos y 35 larvas) y Argia oculata (Selys, 1865) con 46 individuos (43 adultos y 35 larvas). Se considera que el esfuerzo de muestreo fue suficiente debido a que las curvas de acumulación de especies casi alcanzaron las asíntotas con los estimadores (figura 3). Sin embargo, estas curvas de acumulación indican que, aun así, es posible encontrar algunas especies de Odonata que no fueron recolectadas en nuestro período de muestreo.

El ANOVA $(p<0,05 ; \mathrm{F}=0,44)$ no mostró diferencias significativas en el índice de diversidad de Shannon-Wiener entre estaciones de muestreo y períodos. Sin embargo, la Estación 2 y el mes de febrero registraron la mayor diversidad durante todo el año de muestreo (figura 4). Por otro lado, el índice de similitud de Jaccard expuso que las estaciones y períodos de muestreo no comparten las mismas especies, por lo que existen diferencias en la composición 
Tabla 1. Lista de todas las especies de Odonata recolectadas en cada estación y mes de muestreo

\begin{tabular}{|c|c|c|c|c|c|}
\hline \multicolumn{2}{|l|}{ Especie } & \multirow{2}{*}{$\begin{array}{l}\text { Adultos } \\
0\end{array}$} & \multirow{2}{*}{$\begin{array}{l}\text { Larvas } \\
1\end{array}$} & \multirow{2}{*}{$\begin{array}{l}\text { Estación } \\
2\end{array}$} & \multirow{2}{*}{$\begin{array}{l}\text { Mes } \\
\text { Nov }\end{array}$} \\
\hline Aeshnidae & $\begin{array}{l}\text { Coryphaeschna viriditas } \\
\text { Calvert, } 1952\end{array}$ & & & & \\
\hline \multirow{2}{*}{ Calopterygidae } & $\begin{array}{l}\text { Hetaerina caja } \\
\text { Drury, } 1773\end{array}$ & 11 & 0 & 2,4 & May, Nov \\
\hline & $\begin{array}{l}\text { Hetaerina occisa } \\
\text { Selys, } 1853\end{array}$ & 26 & 19 & Todas & Todos \\
\hline \multirow{5}{*}{ Coenagrionidae } & $\begin{array}{l}\text { Acanthagrion kennedii } \\
\text { Williamson, } 1916\end{array}$ & 15 & 1 & $2,3,4$ & Todos \\
\hline & $\begin{array}{l}\text { Argia cupraurea } \\
\text { Calvert, } 1901\end{array}$ & 3 & 0 & 2 & May \\
\hline & $\begin{array}{l}\text { Argia fissa } \\
\text { Selys, } 1865\end{array}$ & 1 & 0 & 1 & Ago \\
\hline & $\begin{array}{l}\text { Argia oculata } \\
\text { Selys, } 1865\end{array}$ & 43 & 3 & Todas & Todos \\
\hline & $\begin{array}{l}\text { Enallagma novaehispaniae } \\
\text { Calvert, } 1907\end{array}$ & 32 & 35 & $2,3,4$ & Todos \\
\hline \multirow{7}{*}{ Libellulidae } & $\begin{array}{l}\text { Dythemis nigra } \\
\text { Meurgey \& Poiron, } 2011\end{array}$ & 4 & 4 & Todas & Todos \\
\hline & $\begin{array}{l}\text { Elasmothemis cannacrioides } \\
\text { Calvert, } 1906\end{array}$ & 0 & 3 & 2 & Feb, May \\
\hline & $\begin{array}{l}\text { Erythrodiplax castanea } \\
\text { Burmeister, } 1839\end{array}$ & 11 & 2 & 3,4 & Todos \\
\hline & $\begin{array}{l}\text { Elga leptostyla } \\
\text { Ris, } 1909\end{array}$ & 0 & 3 & 1,2 & Feb, Nov \\
\hline & $\begin{array}{l}\text { Libellula herculea } \\
\text { Karsh, } 1889\end{array}$ & 1 & 1 & 1 & May, Nov \\
\hline & $\begin{array}{l}\text { Macrothemis hemichlora } \\
\text { Burmeister, } 1839\end{array}$ & 5 & 23 & Todas & Todos \\
\hline & $\begin{array}{l}\text { Perithemis mooma } \\
\text { Kirby, } 1889\end{array}$ & 16 & 5 & $2,3,4$ & Todos \\
\hline Gomphidae & $\begin{array}{l}\text { Erpetogomphus sabaleticus } \\
\text { Williamson, } 1918\end{array}$ & 0 & 4 & 1 & Todos \\
\hline Total & & 168 & 104 & & \\
\hline
\end{tabular}

de especies de Odonata en cada estación y período de muestreo (figura 5).

Finalmente, se encontró una variación significativa en la conductividad y la concentración de nutrientes $\left(\mathrm{NH}_{4}^{+}\right.$y $\left.\mathrm{NO}_{3}^{-}\right)(p<0,05 ; \mathrm{F}=1,65)$ a lo largo del arroyo. Se observó un aumento de casi diez veces en la conductividad que cambió de $14 \mu \mathrm{s} \mathrm{cm}^{-1}$ en la Estación 1 a $144 \mu \mathrm{s} \mathrm{cm}^{-1}$ en la Estación 4 (tabla 2). Los nutrientes también aumentaron: el amonio $\left(\mathrm{NH}_{4}^{+}\right)$ aumentó de 0,06 a 0,31 $\mathrm{mg} \mathrm{dm}^{-3}$, de la Estación 1 a la Estación 3 y el nitrato $\left(\mathrm{NO}_{3}^{-}\right)$aumentó de 0 a $10 \mathrm{mg}$ $\mathrm{L}^{-1}$, a la Estación 4 (tabla 2). En contraste, el fosfato $\left(\mathrm{PO}_{4}^{-3}\right)$ no mostró diferencias significativas entre las estaciones de muestreo. Simultáneamente, las características fisicoquímicas variaron con el tiempo, el $\mathrm{pH}$ y la temperatura, mostrando diferencias significativas a lo largo del año $(p<0,05 ; \mathrm{F}=2,35)$. La temperatura cambió de un valor medio de $24,3{ }^{\circ} \mathrm{C}$ en la estación húmeda, a un máximo valor de 28,37 ${ }^{\circ} \mathrm{C}$ registrado en la estación seca, y el pH osciló entre 7,73 y 10,01, entre la estación seca y la lluviosa (tabla $2)$.

\section{DISCUSIÓN}

La diversidad de los odonatos no cambió significativamente a lo largo de las estaciones ni los periodos de muestreo. Esto es causado por la alta tasa de dispersión y la movilidad en odonatos adultos que generan que las especies compartan diferentes puntos de muestreo (Altamiranda, 2009; Collins y McIntyre, 2015). Del mismo modo, el orden Odonata, tanto larvas como adultos, es un grupo de insectos que no tiene necesidad trófica directa por las macrófitas y 


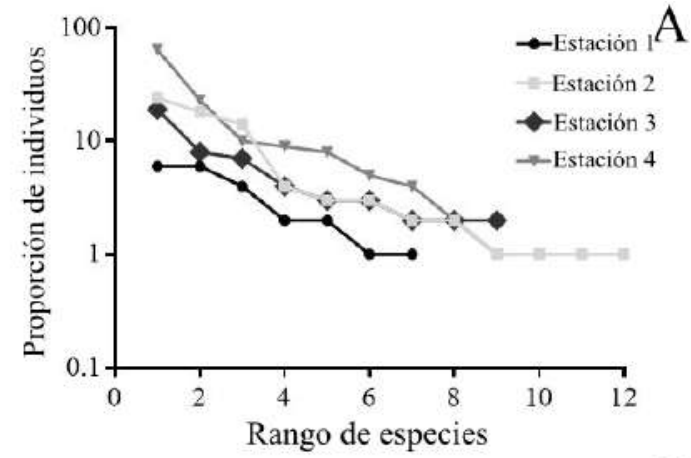

B

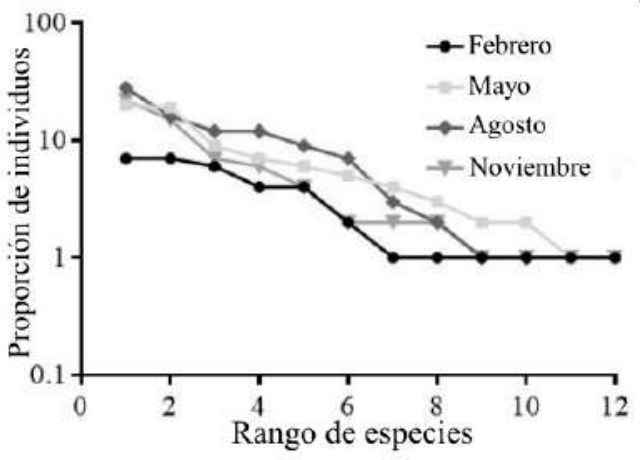

Figura 2. Abundancia de Odonata en cada Estación (A) y periodos (B) de muestreo en el arroyo La Avería en Paicol (Huila-Colombia).

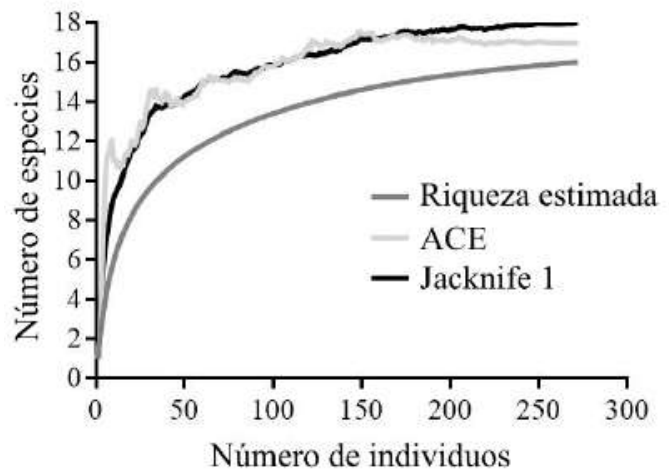

Figura 3. Curvas de acumulación de especies de Odonata en el arroyo La Avería, en Paicol (HuilaColombia). Para el índice ACE se consideraron como especies raras aquellas con 5 o menos individuos recolectados.

plantas ribereñas, por lo que la estructura vegetal riparia no afecta significativamente la distribución de muchas especies de Odonata (Garzón y Realpe, 2009; González-Soriano, Noguera, Zaragoza-Caballero, y Ramírez-García, 2009). No obstante, observamos valores de diversidad más altos en la Estación 2 y la Estación 3, lo que concuerda con otras investigaciones en Colombia y en los trópicos, donde se registra una mayor diversidad de Odonata vinculada con la inter-

Tabla 2. Promedio (B) y desviación estándar (SD) de los datos fisicoquímicos presentes en cada estación de muestreo (estación 1 = áreas conservadas; estación 2, 3 y 4 = áreas perturbadas) y tiempo, en el arroyo La Avería en Paicol (Huila). Tamaño de muestra $(\mathrm{N})=16$

\begin{tabular}{|c|c|c|c|c|c|c|c|c|c|c|c|c|c|c|c|c|}
\hline & Estacic & ón 1 & Estaci & on 2 & Estació & & Estac & ón 4 & Febre & & Mayo & & Agosto & & Novie & nbre \\
\hline Parámetro & \begin{tabular}{l|l}
$\mathrm{B}$ &
\end{tabular} & SD & \begin{tabular}{l|}
$\mathrm{B}$ \\
\end{tabular} & SD & $\mathrm{B}$ & SD & $\mathrm{B}$ & SD & $\mathrm{B}$ & SD & \begin{tabular}{l|l}
$\mathrm{B}$ \\
\end{tabular} & SD & \begin{tabular}{l|l}
$B$ \\
\end{tabular} & SD & $\mathrm{B}$ & SD \\
\hline 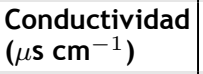 & 14 & 28 & 85.25 & 62.33 & 114.75 & 47.39 & 144 & 58.84 & 107 & 103.35 & 52.75 & 37.41 & 65.75 & 49.92 & 132.5 & 53.03 \\
\hline $\begin{array}{l}\text { Oxígeno } \\
\text { disuelto } \\
\left(\mathrm{mg} \mathrm{L}^{-1}\right)\end{array}$ & 7.24 & 0.2 & 7.88 & 0.3 & 18.32 & 2.1 & 7.17 & 0.71 & 6.02 & 3.04 & 6.78 & 0.9 & 6.73 & 1.28 & 7.35 & 0.87 \\
\hline $\begin{array}{l}\text { SST } \\
\left(\mathrm{mg} \mathrm{L}^{-1}\right)\end{array}$ & 7 & 14 & 40 & 26.27 & 230 & 23.85 & 71.5 & 28.9 & 53.25 & 51.19 & 26.25 & 18.55 & 32.75 & 24.97 & 63.75 & 24.01 \\
\hline $\mathrm{pH}$ & 6.98 & 0.49 & 7.59 & 1.13 & 31.12 & 1.52 & 7.63 & 1.29 & 7.55 & 0.14 & 6.74 & 0.38 & 6.77 & 0.4 & 8.92 & 1.24 \\
\hline $\begin{array}{l}\text { Temperatura } \\
\left({ }^{\circ} \mathrm{C}\right)\end{array}$ & 24.82 & 0.86 & 25.38 & 1.13 & 103.27 & 2.1 & 25.1 & 1.2 & 26.71 & 1.26 & 23.85 & 0.26 & 24.6 & 0.22 & 25.95 & 0.51 \\
\hline $\begin{array}{l}\text { Caudal } \\
\left(\mathrm{m}^{3} \mathrm{~s}^{-1}\right)\end{array}$ & 0.39 & 0.23 & 1.04 & 0.3 & 3.1 & 0.34 & 1.16 & 0.75 & 0.41 & 0.18 & 0.78 & 0.43 & 1.01 & 0.04 & 1.17 & 0.77 \\
\hline $\begin{array}{l}\mathrm{NH}_{4}^{+} \\
\left(\mathbf{m g ~ L}^{-1}\right)\end{array}$ & 0.06 & 0.13 & 0.19 & 0.13 & 12.5 & 1.75 & 0.31 & 0.13 & 1.19 & 1.89 & 1.06 & 1.96 & 1.13 & 1.92 & 0.31 & 0.13 \\
\hline $\begin{array}{l}\mathrm{NO}_{2}^{-} \\
\left(\mathrm{mg} \mathrm{L}^{-1}\right)\end{array}$ & 0 & 0 & 1.25 & 2.5 & 5 & 2.5 & 10 & 7.07 & 2.5 & 2.89 & 6.25 & 9.46 & 2.5 & 5 & 1.25 & 2.5 \\
\hline $\begin{array}{l}\mathrm{PO}_{4}^{-3} \\
\left(\mathrm{mg} \mathrm{L}^{-1}\right)\end{array}$ & 0.13 & 0.25 & 0.25 & 0 & 2.75 & 0.38 & 1.31 & 0.85 & 0.94 & 0.77 & 0.56 & 0.52 & 0.69 & 0.9 & 0.19 & 0.13 \\
\hline
\end{tabular}


vención antrópica (Altamiranda, 2009; Hernandez, Reece, y McIntyre, 2006; Novelo-Gutiérrez y GómezAnaya, 2009; Urrutia, 2005). Los valores más altos de diversidad de Odonata en entornos perturbados se asocian con una mayor abundancia de macrófitas, que proporcionan hábitats para la alimentación y la reproducción en especies con alta capacidad de dispersión y bajos requisitos ecológicos (Brix, 1997; Hernandez et al, 2006; Miserendino y Masi, 2010; Perron y Pick, 2019). En un caso similar, se registran los datos de diversidad más altos durante la estación húmeda (febrero). Estos valores de mayor diversidad están relacionados con la abundante oferta de hábitats y presas, como mosquitos y moscas, causados por más precipitaciones durante la temporada de lluvias (Ball-Damerow, M'Gonigle, y Resh, 2014; Janzen y Schoener, 1968; Vilela, Guillermo-Ferreira, y Del-Claro, 2016). Finalmente, se podría inferir que los parches de bosque de la zona estudiada son demasiado pequeños para albergar una comunidad madura de odonatos. Por esa razón, se recomienda extender el área y tiempo de muestreo en futuras investigaciones.
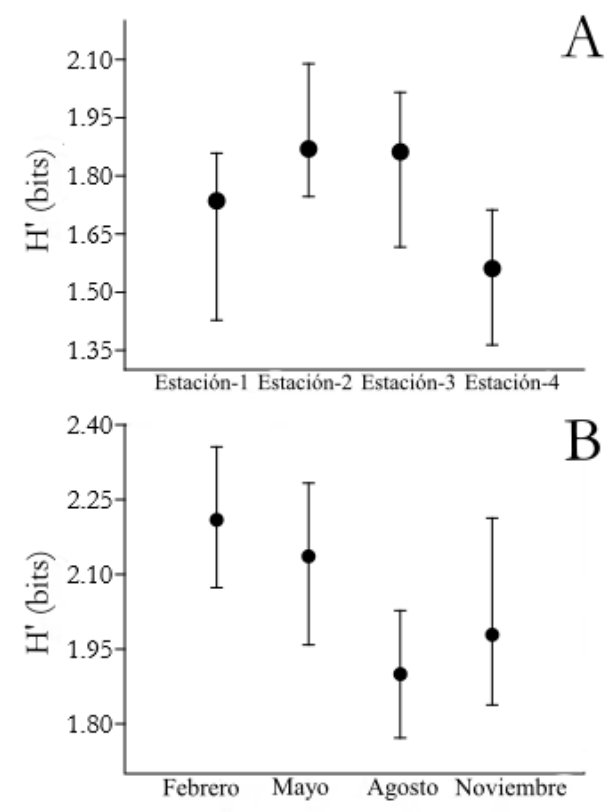

Figura 4. Variación en el índice de diversidad Shannon-Wiener $\left(\mathrm{H}^{\prime}\right)$ con el error estándar para los odonatos colectados, tanto adultos como larvas, por Estación (A) y periodo de muestreo (B).
Se encontraron diferencias en la composición de Odonata entre estaciones y períodos de muestreo. Estos cambios se producen por el reemplazo vegetal en el bosque ripario nativo hacia sistemas de cultivos, arbustos y macrófitas, como se detalla a lo largo del arroyo (Monteiro-Júnior et al, 2013). Hay especies de Odonata adaptadas a áreas perturbadas (cultivos de arroz y áreas de ganado), como es el caso de Acanthagrion kennedii, Enallagma novaehispaniae, Erythrodiplax castanea, Hetaerina caja y Perithemis mooma, especies que prefieren áreas abiertas con una alta densidad de macrófitas, una característica registrada solo en las partes bajas del sistema lótico (Estaciones 3 y 4) (Altamiranda, 2009; MonteiroJúnior et al, 2013; Rache-Rodríguez, 2015; Suhonen, Korkeamäki, Salmela, y Kuitunen, 2014).

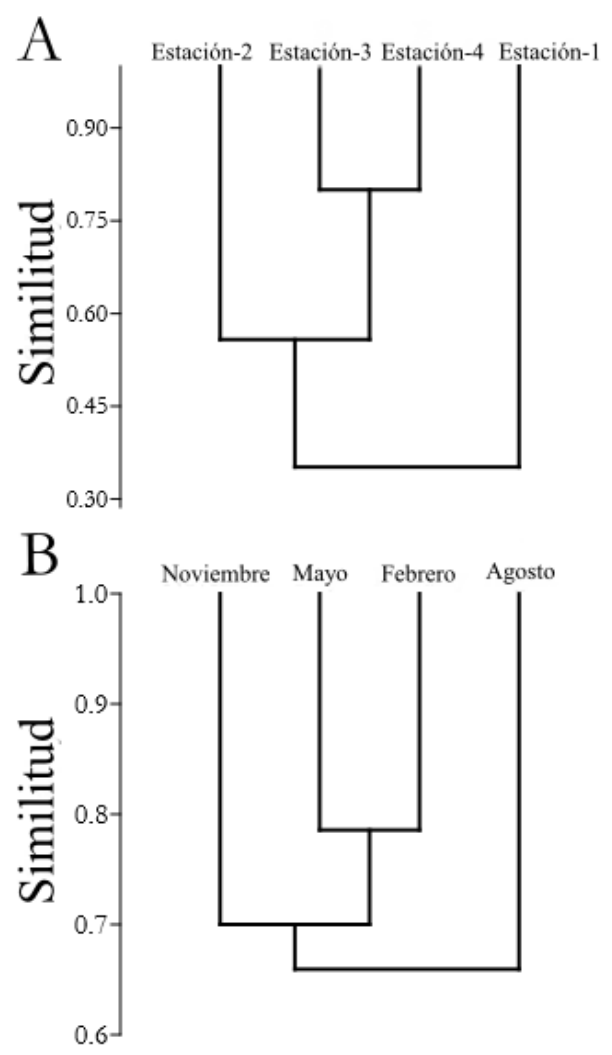

Figura 5. Especies de Odonata, tanto adultos como larvas, definidos de acuerdo a grupos de similitud por medio del índice de Jaccard y el algoritmo paired-group, entre las estaciones $(\mathbf{A})$ y los períodos de muestreo (B). 
De igual manera, hubo cuatro especies que encontramos a lo largo de todo el arroyo: Argia oculata, Dythemis nigra, Hetaerina occisa y Macrothemis hemichlora. Estas especies se caracterizan por una alta movilidad y ausencia de preferencias por el tipo de vegetación (Altamiranda, 2009; Renner, Périco, Schmidt-Dalzochio, y Sahlen, 2018; Simaika, Samways, y Frenzel, 2016).

Con respecto a los cambios significativos en las variables fisicoquímicas a lo largo del arroyo, se observó la influencia de los cultivos de arroz y el vertimiento de residuos urbanos en la Estación 3, que promueven el aumento de las variables de conductividad y nutrientes, especialmente amonio y nitrato (GarcíaVelázquez y Gallardo, 2017; Ometo et al, 2000). Esta alteración en las características del agua podría estar relacionada con cambios en la distribución de macrófitas y la diversidad de larvas de Odonata a lo largo del arroyo (Brix, 1997; Kasangaki, Chapman, y Balirwa, 2008; Perron y Pick, 2019); sin embargo, en este estudio no se obtuvieron datos suficientes para explicar esta correlación.

En conclusión, dados los cambios en la riqueza y abundancia de Odonata entre estaciones de muestreo y períodos, se acepta en parte la hipótesis previamente planteada Estos cambios están causados por el cambio en la cobertura vegetal riparia que promueven la presencia de diferentes especies de Odonata a lo largo del arroyo. No obstante, la diversidad de Odonata no cambia a lo largo del arroyo, debido a que estos requisitos ecológicos no son tan relevantes en la distribución de estos insectos. Finalmente, consideramos este estudio como un paso preliminar para una mayor valoración ecológica en los ecosistemas acuáticos de Colombia, especialmente en las regiones BST donde el ecosistema ripario se ha estado fragmentado. Estos resultados resaltan la necesidad de más estudios de Odonata en los parches remanentes de BST en Huila y en Colombia.

\section{AGRADECIMIENTOS}

Agradecemos al Laboratorio de Ecología y Biodiversidad de la Universidad Militar Nueva Granada por el préstamo de equipos. Agradecemos a Alonso Ramírez por brindar apoyo en el diseño y análisis del experimento. Agradecemos al grupo de investigación LAZOEA de la Universidad de los Andes, por su apoyo con la identificación de las muestras. Agradecemos a la Alcaldía Municipal de Paicol, por proporcionar mapas e información climática. Agradecemos especialmente a Norberto Cuéllar, Aida Cardozo, Santiago Díaz, José Cifuentes y Andrés Mafla por su invaluable ayuda en el campo.

\section{CONFLICTO DE INTERESES}

Los autores declaran que no existe ningún conflicto de interés con la investigación presentada.

\section{REFERENCIAS}

Altamiranda, M. (2009). Diversidad de Libélulas (InsectaOdonata) para dos Usos de Suelo, en un Bosque Seco Tropical. Revista Facultad Nacional Agronomía Medellin, 62(2), 50715079. Recuperado de: http: //www.scielo.org.co/scielo.php?script=sci_arttext\& pid $=$ S0304-28472009000200006\&lng $=$ en\&nrm $=$ iso. ISSN 0304-2847

Ball-Damerow, J.E., M'Gonigle, L.K., and Resh, V.H. (2014). Local and regional factors influencing assemblages of dragonflies and damselflies (Odonata) in California and Nevada. Journal of Insect Conservation, 18(6), 10271036. DOI:10.1007/s10841-014-9709-6

Belle, J. (1996). Higher classification of the SouthAmerican Gomphidae (Odonata). Zoologische Mededelingen, 70(21), 297-324.

Benke, A.C., Huryn, A.D., Smock, L.A, and Wallace, J.B. (1999). Length-mass relationships for freshwater macroinvertebrates in North America with particular reference to the southeastern United States. Journal of the North American Benthological Society, 18(3), 308343. DOI: $10.2307 / 1468447$

Brix, H. (1997). Do macrophytes play a role in constructed wetlands?. Water Science and Technology, 35(5), 11-17. DOI:10.1016/S0273-1223(97)00047-4

Carvajal-Cogollo, J.E. (2008). Patrones de diversidad y composición de reptiles en fragmentos de bosque seco tropical en Córdoba, Colombia. Tropical Conservation Science, 1(4), 397416. https://tropicalconservationscience. mongabay.com/content/v1/08-12-01-Carvajal-Cogollo_ and_Urbina-Cardona_397-416.pdf

Collins, S.D., and McIntyre, N.E. (2015). Modeling the distribution of odonates: a review. Freshwater Science, 34(3), 11441158. DOI:10.1086/682688

Colwell, R.K., Chao, A., Gotelli, N.J., Lin, S.Y., Mao, C.X., Chazdon, R.L., and Longino, J.T. (2012). Models and estimators linking individual-based and samplebased rarefaction, extrapolation and comparison of assemblages. Journal of Plant Ecology, 5(1), 321. DOI:10.1093/jpe/rtr044 
Corbet, P.S. (2004). Dragonflies Behaviour and Ecology of Odonata (Revised Edition). (P. S. Corbet, Ed.) (Harley Boo). Colchester: Harley Books.

De Marmels, J. (2012). Review of the "metallic group" of species of Argia Rambur known from Venezuela, with description of the larva of Argia jocosa Hagen in Selys, 1865 (Odonata: Coenagrionidae). International Journal of Odonatology, 15(3), 249262. DOI:10.1080/13887890.2012.717906

Feld, C., Birk, S., Eme, D., Gerisch, M., Hering, D., Kernan, M., Maileht, K, Mischke U, Ott I, Pletterbauer F, Poikane S, Salgado J, Sayer C, van Wichelen J, and Malard, F. (2016). Disentangling the effects of land use and geo-climatic factors on diversity in European freshwater ecosystems. Ecological Indicators, 60, 7183. DOI:10.1016/j.ecolind.2015.06.024

Firmiano, K.R., Ligeiro, R., Macedo, D.R., Juen, L., Hughes, R.M., and Callisto, M. (2017). Mayfly bioindicator thresholds for several anthropogenic disturbances in neotropical savanna streams. Ecological Indicators, 74, 276284. DOI:10.1016/j.ecolind.2015.06.024

García-Velázquez, L., and Gallardo, A. (2017). El ciclo global del nitrógeno. Una visión para el ecólogo terrestre. Ecosistemas, 26(1), 46. DOI:.7818/ECOS.2017.26-1.02

Garrison, R.W. (1994). A revision of the New World genus Erpetogomphus Hagen in Selys (Odonata: Gomphidae). Tijdschrift Voor Entomologie, 137(2), 173269.

Garrison, R.W., and Ellenrieder, N. Von. (2015). Damselflies of the genus Argia of the Guiana Shield (Odonata: Coenagrionidae). Zootaxa, 4042(1), 1-134. DOI:10.11646/zootaxa.4042.1.1

Garrison, R.W., Ellenrieder, N. von, and Louton, J.A. (2006). Dragonfly Genera of the New World. Baltimore: The Johns Hopkins University Press.

Garzón, C., y Realpe, E. (2009). Diversidad de Odonata (Insecta) en la reserva natural Cabildo Verde (Sabana de Torres, Santander, Colombia), una aproximación hacia la conservación. Caldasia, 31(2), 459470. https://revistas. unal.edu.co/index.php/cal/article/view/36132/37571

Gómez, M.F., Moreno, L.A., Andrade, G.I., y Rueda, C. (2016). BIODIVERSIDAD 2015: Estado y tendencias de la biodiversidad continental de Colombia. Bogotá: Instituto Alexander von Humboldt (IAvH). DOI:10.1017/CBO9781107415324.004

González-Soriano, E., Noguera, F.A., Zaragoza-Caballero, S., y Ramírez-García, E. (2009). Odonata de un bosque tropical caducifolio: sierra de San Javier , Sonora , México. Revista Mexicana De Biodiversidad, 80, 341348. http://www.scielo.org.mx/scielo.php? script $=$ sci_arttext\&pid $=$ S1870-34532009000200009

Hammer, Ø., Harper, D.A.T., and Ryan, P.D. (2001). PAST: Paleontological Statistics Software Package for Education and Data Analysis. Palaeontologia Electrónica, 4(1), 19. DOI:10.1016/j.bcp.2008.05.025

Hepp, L.U., and Santos, S. (2009). Benthic communities of streams related to different land uses in a hydrographic basin in southern Brazil. Environmental Monitoring and Assessment, 157(14), 305318. DOI:10.1007/s10661-0080536-7

Hernandez, K.M., Reece, B.A., and McIntyre, N.E. (2006). Effects of Anthropogenic Land Use on Odonata in
Playas of the Southern High Plains. Western North American Naturalist, 66(3), 273278. DOI:10.3398/15270904(2006)66[273:EOALUO]2.0.CO;2

Instituto de Hidrología, Meteorología y Estudios Ambientales. (2016). http://www.ideam.gov.co/ solicitud-de-informacion

Janzen, D.H., and Schoener, T.W. (1968). Differences in insect abundance and diversity between wetter and drier sites during a tropical dry season. Ecology, 49(1), 96110. DOI: $10.2307 / 1933565$

Jackson, D.A., and Somers, K.M. (1989). Are probability tests from the permutation model of Mantel's test stable? Canadian Journal of Zoology, 67(3), 766-769. DOI:10.1139/z89-108

Johnson, R.C., Jin, H.S., Carreiro, M.M., and Jack, J.D. (2013). Macroinvertebrate community structure, secondary production and trophic-level dynamics in urban streams affected by non-point-source pollution. Freshwater Biology, 58(5), 843857. DOI:10.1111/fwb.12090

Kasangaki, A., Chapman, L.J., and Balirwa, J. (2008). Land use and the ecology of benthic macroinvertebrate assemblages of high-altitude rainforest streams in Uganda. Freshwater Biology, 53(4), 681697. DOI:10.1111/j.13652427.2007.01925.x

Kutcher, T.E., and Bried, J.T. (2014). Adult Odonata conservatism as an indicator of freshwater wetland condition. Ecological Indicators, 38, 3139. DOI:10.1016/j.ecolind.2013.10.028

Leonard, J.W. (1977). A revisionary study of the genus Acanthagrion (Odonata: Zygoptera). Miscellaneous Publications Museum of Zoology, University of Michigan, (153), 188. Retrieved from http://deepblue.lib.umich. $\mathrm{edu} /$ handle/2027.42/56397

Limongi, S.J. (1983). Estudio Morfo-taxonomico de Náyades en algunas especies de Odonata (Insecta) en Venezuela (I). Universidad Central de Venezuela.

May, M.L. (1998). Macrothemis Fallax , a new species of dragonfly from Central America (Anisoptera: Libellulidae), with a key to male Macrothemis. International Journal of Odonatology, 1(2), 137153. DOI:10.1080/13887890.1998.9748101

McGeogh, M.A. (1997). The selection, testing and application of terrestrial insects as bioindicators. Biological Reviews, 73(2), 181201. DOI:10.1111/j.1469-185X.1997.tb00029.x

Meurgey, F., and Poiron, C. (2011). The true Dythemis multipunctata Kirby, 1894, from the West Indies and proposed new taxonomic status (Odonata: Anisoptera: Libellulidae). Zootaxa, 3019, 5162. DOI:10.11646/zootaxa.3019.1.3

Miles, L., Newton, A.C., DeFries, R.S., Ravilious, C., May, I., Blyth, S., Kapos, V., and Gordon, J.E. (2006). A global overview of the conservation status of tropical dry forests. Journal of Biogeography, 33(3), 491505. DOI:10.1111/j.1365-2699.2005.01424.x

Miserendino, M.L., and Masi, C.I. (2010). The effects of land use on environmental features and functional organization of macroinvertebrate communities in Patagonian low order streams. Ecological Indicators, 10(2), 311319. DOI:10.1016/j.ecolind.2009.06.008

Monteiro-Júnior, C. da S., Couceiro, S.R.M., Hamada, N., and 
Juen, L. (2013). Effect of vegetation removal for road building on richness and composition of Odonata communities in Amazonia, Brazil. International Journal of Odonatology, 16(2), 11. DOI:10.1080/13887890.2013.764798

Montenegro, O.L. (2009). La conservación biológica y su perspectiva evolutiva. Acta Biológica Colombiana, 14, 255268. https://revistas.unal.edu.co/index.php/ actabiol/article/view/11578/20610

Moulton, T.P. (2006). Why the world is green, the waters are blue and foodwebs in small streams in the atlantic rainforest are predominantly driven by microalgae? Oecologia Brasiliensis, 10(01), 7889. DOI:10.4257/oeco.2006.1001.05

Neres-Lima, V., Machado-Silva, F., Baptista, D.F., Oliveira, R.B.S., Andrade, P.M., Oliveira, A.F., Sasada-Sato, C.Y., Silva-Junior E.F., Feijó-Lima, R, Angelini, R., Camargo, P,B., and Moulton, T.P. (2017). Allochthonous and autochthonous carbon flows in food webs of tropical forest streams. Freshwater Biology, 62 (6), 10121023. DOI:10.1111/fwb.12921

Novelo-Gutiérrez, R. (2002). Descripción de las larvas de Perithemis intensa Kirby, 1889 y P. domitia (Drury, 1773), con notas sobre otras larvas del género en México (Odonata: Anisoptera: Libellulidae). Folia Entomológica Mexicana, 41(3), 321327. Recuperado de http://biblat.unam.mx/pt/revista/folia-entomologicamexicana/articulo/descripcion-de-las-larvas-deperithemis-intensa-kirby-1889-y-p-domitia-drury-1773con-notas-sobre-otras-larvas-del-genero-en-mexicoodonata-anisoptera-libellulidae

Novelo-Gutierrez, R. (2005). La larva de Enallagma novaehispaniae Calvert, 1902 (Odonata: Zygoptera: Coenagrionidae). Folia Entomológica Mexicana, 44(2), 219224. Recuperado de http://www.redalyc.org/articulo.oa?id= 42444210

Novelo-Gutiérrez, R., and Gómez-Anaya, J.A. (2009). A comparative study of Odonata (Insecta) assemblages along an altitudinal gradient in the sierra de Coalcomán Mountains, Michoacán, Mexico. Biodiversity and Conservation, 18(3), 679698. DOI:10.1007/s10531-008-9533-y

Ometo, J.P., Martinelli, L.A., Ballester, M.V., Gessner, A., Krusche, A.V., Victoria, R.L., and Williams, M. (2000). Effects of land use on water chemistry and macroinvertebrates in two streams of the Piracicaba river basin, south-east Brazil. Freshwater Biology, 44(2), 327337. DOI:10.1046/j.1365-2427.2000.00557.x

Palacino-Rodríguez, F. (2016). Two decades of progress in over one hundred years of study: Present status of Odonata research in Colombia. Odonatologica, 45(3-4): 327. DOI:10.5281/zenodo.163455

Palmer, M.W. (1990). The Estimation of Species Richness by Extrapolation. Ecology, $71(3), 11951198$. DOI:10.2307/1937387

Perron, M.A.C., and Pick, F.R. (2019). Stormwater ponds as habitat for Odonata in urban areas: the importance of obligate wetland plant species. Biodiversity and Conservation, 29(3). DOI:10.1007/s10531-019-01917-2

Pizano, C., y Garcia, H. (2014). El Bosque Seco Tropical en Colombia. Instituto de Investigacion Alexander von Humbolt (Primera Ed). Bogotá: Instituto Alexander von Hum- boldt (IAvH). DOI:10.1017/CBO9781107415324.004

Rache-Rodríguez, L. (2015). Caracterización de hábitat y morfología de algunas especies del género Perithemis (Odonata: Anisoptera) presentes en la Cordillera Oriental. Universidad Nacional de Colombia. Recuperado de http: //www.bdigital.unal.edu.co/52351/

Renner, S., Périco, E., Schmidt-Dalzochio, M., and Sahlen, G. (2018). Water body type and land cover shape the dragonfly communities (Odonata) Water body type and land cover shape the dragonfly communities (Odonata) in the Pampa biome, Rio Grande do Sul, Brazil. Journal of Insect Conservation, 22(0), 113-125. DOI:10.1007/s10841017-0042-8

Ris, F. (1930). A revision of the Libelluline genus Perithemis (Odonata). Miscellaneous Publications University of Michigan Museum of Zoology. Recuperado de http: //deepblue.lib.umich.edu/handle/2027.42/56266

Silva, D.D.P., De Marco, P., and Resende, D.C. (2010). Adult odonate abundance and community assemblage measures as indicators of stream ecological integrity: A case study. Ecological Indicators, 10, 744752. DOI:10.1016/j.ecolind.2009.12.004

Simaika, J.P., Samways, M.J., and Frenzel, P.P. (2016). Artificial ponds increase local dragonfly diversity in a global biodiversity hotspot. Biodiversity and Conservation, 25(10), 19211935. DOI:10.1007/s10531-016-1168-9

Suhonen, J., Korkeamäki, E., Salmela, J., and Kuitunen, M. (2014). Risk of local extinction of Odonata freshwater habitat generalists and specialists. Conservation Biology, 28(3), 783789. DOI:10.1111/cobi.12231

Tognelli, M.F., Lasso, C.A., Bota-Sierra, C.A., JiménezSegura, L.F., y Cox, N.A. (2016). Estado de conservación y distribución de la biodiversidad de agua dulce en los andes tropicales. (M. F. Tognelli, C. A. Lasso, C. A. Botasierra, L. F. Jiménez-segura, y N. A. Cox, Eds.) (UICN). Gland, Cambridge y Arlington: UICN.

Urrutia, M.X. (2005). Riqueza de especies de Odonata Zigoptera por unidades fisiográficas en el departamento del Valle del Cauca. Boletín Del Museo de Entomología de La Universidad Del Valle, 6(2), 3036. DOI:10.1017/CBO9781107415324.004

Valdés, A. (2011). Modelos de paisaje y análisis de fragmentación: de la biogeografía de islas a la aproximación de paisaje continuo. Ecosistemas, 20(2), 1120. http://hdl. handle.net/10045/20731

Vargas, W. (2015). A brief description of the vegetation, with special emphasis on the intermediate pioneers of the dry forests of La Jagua, in the upper basin of the Magdalena River in Huila. Colombia Forestal, 18(1), 4770. DOI:10.14483/udistrital.jour.colomb.for.2015.1.a03

Vilela, D.S., Guillermo-Ferreira, R., and Del-Claro, K. (2016). The Odonata community of a brazilian vereda: seasonal patterns, species diversity and rarity in a palm swamp enviroment. BioScience Journal, 32(2), 486495. DOI:10.14393/BJ-v32n2a2016-30491

von Ellenrieder, N. (2003). A synopsis of the Neotropical species of "Aeshna" Fabricius: the genus Rhionaeschna Förster (Odonata: Aeshnidae). Tijdschrift Voor Entomologie, 146(1), 67207. DOI:10.1163/22119434-900000120

Walker, E.M. (2015). A Revision of the Libelluline 
Genus Erythrodiplax (Odonata). Annals of the Entomological Society of America, 36(3), 587588. DOI:10.1093/aesa/36.3.587

Williams-Linera, G., Manson, R.H., e Isunza, E. (2002). La fragmentación del bosque mesófilo de montaña y patrones de uso del suelo en la región oeste de Xalapa, Veracruz, México. Madera y Bosques, 8(1), 7389. Retrieved from http://scholar. google.com/scholar?hl=enybtnG $=$ Search\&q=intitle: La+fragmentaci?n+del+bosque+mes?filo+de+monta? $\mathrm{a}+\mathrm{y}+$ patrones + de+uso+del+suelo+en+la+regi?n+ oeste+de+Xalapa,++ Veracruz,++ M?xico\#0

Zloty, J., Pritchard, G., and Esquivel, C. (1993). Larvae of the Costa Rican Hetaerina (Odonata: Calopterygidae) with comments on distribution. Systematic Entomology, 18, 253265. DOI:10.1111/j.1365-3113.1993.tb00665.x 